\title{
Perspectives on sustainable management of the Poso Lake (Indonesia) endemic ricefish, Oryzias nigrimas (Actinopterygii: Adrianichthyidae)
}

\author{
Novalina Serdiati $^{1 *}$, Diana Arfiati ${ }^{2}$, Maheno Sri Widodo $^{2}$, Tri Djoko Lelono ${ }^{3}$, Samliok Ndobe $^{1}$, \\ Kasim Mansyur ${ }^{1} \&$ Abigail Mary Moore ${ }^{4}$ \\ 1. Aquaculture Study Program, Tadulako University, Palu, Central Sulawesi, Indonesia; novalinaserdiati@untad.ac.id, \\ samliok@untad.ac.id, achim_dive9@yahoo.com \\ 2. Department of Aquatic Resources Management, Brawijaya University, Malang, Indonesia; d-arfiati@ub.ac.id, \\ lynxpardel@yahoo.co.id \\ 3. Department of Utilization of Fisheries and Marine Resources, Brawijaya University, Malang, Indonesia; \\ t.djoko@ub.ac.id \\ 4. Postgraduate School, Hasanuddin University, Makassar, South Sulawesi, Indonesia; abigail@pasca.unhas.ac.id \\ * Correspondence
}

\section{Received 22-VI-2020. Corrected 28-X-2020. Accepted 09-XI-2020.}

\begin{abstract}
Introduction: The endemic fishes of the ancient lakes of Sulawesi are under increasing threat. Objective: To evaluate the data and information available from a holistic management perspective and to formulate measures to conserve the endemic ricefish Oryzias nigrimas in Poso Lake, Indonesia. Methods: Collection of primary data from three stations around Lake Poso and literature study. Results: Threats to O. nigrimas include habitat degradation and loss, introduced alien species, and exploitation as a locally important food fish. Options to promote sustainable fisheries management include spatial and temporal limitations to minimise catch of gravid or brooding fish. Habitat protection should include measures to minimise impacts from activities which can reduce water quality and disturb or kill aquatic vegetation. Conclusion: Measures to prevent further $O$. nigrimas population decline are considered urgent and further research is recommended to fill identified knowledge gaps. Ex-situ conservation, including the development of captive breeding, could also contribute to a holistic $O$. nigrimas conservation strategy.
\end{abstract}

Key words: endemism; lacustrine; Oryziinae; black buntingi; invasive species; light fishing.

Serdiati, N., Arfiati, D., Widodo, M.S., Lelono, T.D., Ndobe, S., Mansyur, K., \& Moore, A.M. (2021). Perspectives on sustainable management of the Poso Lake (Indonesia) endemic ricefish, Oryzias nigrimas (Actinopterygii: Adrianichthyidae). Revista de Biología Tropical, 69(1), 139-152. DOI 10.15517/rbt.v69i1.42404

Sulawesi Island, the largest landmass in the Wallacea region, is renowned for high levels of endemicity, including aquatic taxa (Hadiaty, 2018; von Rintelen \& Cai, 2009; von Rintelen, Stelbrink, Marwoto, \& Glaubrecht, 2014). The "ancient lakes" of Sulawesi, including the Malili Lake complex in South Sulawesi and Poso Lake in Central Sulawesi, are especially rich in endemic fishes and invertebrates (Meisner, 2001; von Rintelen \& Glaubrecht,
2006; Meixner et al., 2007; Schubart \& Ng, 2008; Walter, Hogan, Haffner, \& Heath, 2011; von Rintelen \& Cai, 2009; Mokodongan \& Yamahira, 2015; Vaillant, Bock, Haffner, \& Cristescu, 2013; von Rintelen et al., 2014)

The ricefishes (Adrianichthyidae) are a family with 33 recognised (valid) species in 2013 (Kottelat, 2013), a number which has since increased to at least 37 (Mandagi, Mokodongan, Tanaka, \& Yamahira, 2018), including the 
description of two new species from Sulawesi: Oryzias soerotoi (Mokodongan, Tanaka, \& Yamahira, 2014); Oryzias dopingdopingensis (Mandagi et al., 2018). Of these 37 species, 21 ricefishes are native to Sulawesi, a recognised "hotspot" of biodiversity for this fish family (Parenti, 2008; Mokodongan et al., 2014; Hadiaty, 2018; Mandagi et al., 2018). While Oryzias javanicus is widely distributed across Southeast Asia (Mokodongan, 2019a), and $O$. celebensis (previously considered endemic to Sulawesi alone has also been reported from East Timor (Parenti, 2008; Hadiaty, 2018; Lumbantobing, 2019a), the majority (19 species or $90.5 \%$ ) of the Sulawesi ricefishes are endemic to one or more of the ancient lakes and/or certain riverine systems in Sulawesi (Parenti, 2008; Hadiaty, 2018; Mandagi et al., 2018; Lumbantobing, 2019b; Lumbantobing, 2019c; Lumbantobing, 2019d; Mokodongan, 2019b; Mokodongan, 2019c; Mokodongan, 2019d; Mokodongan, 2019e; Mokodongan, 2019f; Mokodongan, 2019g; Mokodongan, 2019h; Mokodongan, 2019i; Mokodongan, 2019j; Mokodongan, 2019k; Mokodongan, 20191; Mokodongan, 2019m; Mokodongan, 2019n; Mokodongan, 2019o; Mokodongan, 2019p; Mokodongan, 2019q).

Species with such restricted natural ranges are intrinsically vulnerable to extinction (Walter et al., 2011). The 2019 version of The IUCN Red List of Endangered Species ${ }^{\mathrm{TM}}$ (hereafter referred to as the IUCN Red List) provides assessments of the conservation status according to the criteria of the International Union for the Conservation of Nature (IUCN) for 36 ricefishes (Family Adrianichthyidae), including all but one ( $O$. dopingdopingensis) of the 21 ricefishes reported from Sulawesi. One Sulawesi endemic $(O$. wolasi) is classed as Data Deficient (DD), meaning the data available are insufficient to assign a conservation status.

Globally, half of the assessed ricefishes are classed within one of the IUCN Red List categories indicating a significant risk of extinction; it is remarkable that $89 \%$ of these are ricefishes endemic to Sulawesi. A third of all assessed ricefishes are classed as
Least Concern (LC) including the Poso Lake endemic Xenopoecilus oophorus (synonyms Adrianichthys oophorus and Oryzias oophorus) (Mokodongan, 2019m) and O. celebensis (Lumbantobing, 2019a). However, concerns have been expressed regarding the threats to and urgent need for conservation of all Sulawesi endemic freshwater fishes (Parenti, 2011), including X. oophorus (Gundo, 2010).

Among the ancient lakes of Sulawesi, the ichthyofauna of Lake Poso is particularly rich in ricefishes with seven endemic species (Hadiaty, 2018). The taxonomy of ricefishes at the genus and species levels is a matter of ongoing research (Mokodongan et al., 2018; Parenti, 2008), with several synonyms in current use for some species (e.g. X. oophorus). Using the taxonomy according to Kottelat (2013), the ricefishes recorded in Poso Lake are: Oryzias nigrimas (Parenti, 2008; Serdiati, Arfiati, Widodo, Lelono, \& Gosari, 2019a; Serdiati et al., 2020), O. nebulosus (Parenti \& Soeroto, 2004; Serdiati, Arfiati, Widodo, Lelono, \& Toha, 2019b), O. orthognathus (Parenti \& Soeroto, 2004), Xenopoecilus oophorus and $X$. poptae (Parenti, 2008), Adrianichthys kruyti (Parenti, 2008), and A. roseni (Parenti \& Soeroto, 2004).

Anthropogenic activities pose many direct and indirect threats to the environment in and around Poso Lake (Nursangaji et al., 2014; Mamondol, 2018), Despite their small size, several ricefishes are exploited as food fish (Gundo, 2010; Parenti \& Soeroto, 2004), while the remainder may well be caught as bycatch in targeted (ricefish) and other fisheries. Sulawesi ricefishes are also being proposed as potential model organisms for research in fields as diverse as the study of evolution, behavioural, biological and medical research (Mokodongan \& Yamahira, 2015; Mokodongan et al., 2018; Sari, Andriani, \& Yaqin, 2018; Hilgers \& Schwarzer, 2019; Sutra et al., 2019). Seven of the eight ricefish species assessed as Near Threatened (NT) in the 2019 IUCN Red List are endemic to Sulawesi, and three to Lake Poso: O. nebulosus (Mokodongan, 2019g), 
O. orthognathus (Mokodongan, 2019h), and $O$. nigrimas (Mokodongan, 2019n).

The purpose of this study was to evaluate the Poso endemic ricefish $O$. nigrimas from a holistic conservation management perspective. Based on primary survey data combined with secondary data and information, the study aimed to provide guidance on potential management measures to sustain this species.

\section{MATERIALS AND METHODS}

Field study site: Poso lake (Fig. 1) is situated in Poso District, Central Sulawesi Province, Indonesia (1 ${ }^{\circ} 41^{\prime} 18.42^{\prime \prime}-2^{\circ} 18^{\prime} 3.41^{\prime \prime}$ S \& $120^{\circ} 21^{\prime} 27.10^{\prime \prime}-120^{\circ} 51$ '9.28" E) at an altitude of approximately $657 \mathrm{~m}$ above sea level (Nontji, 2016). Based on measurements made in 2007 (Lukman \& Ridwansyah, 2009), Poso Lake covers an area of $368.9 \mathrm{~km}^{2}$ (36 $890 \mathrm{ha}$ ), with a shoreline of $127 \mathrm{~km}$, length and width of approximately $35.9 \mathrm{~km}$ and $15.3 \mathrm{~km}$. The narrow shallow shelf around the shore reaches depths of around 5-7 $\mathrm{m}$ then plunges steeply to a maximum depth of $\approx 385 \mathrm{~m}$, with a mean depth of $194.7 \mathrm{~m}$ and nearly $2 \mathrm{~m}$ fluctuation in water level between rainy and dry seasons.

Data collection: Primary data were collected primarily at three survey stations around Poso Lake (Fig. 1) on a monthly basis from May 2017 to April 2018. These sites were selected based on information from local fishermen and data from previous surveys. Quantitative in situ measurements visibility (m, Secchi disc), dissolved oxygen (DO) (mg/l, Lutron DO-5510) temperature $\left({ }^{\circ} \mathrm{C}\right)$ and $\mathrm{pH}$ (ATC Tri-meter) were supplemented by qualitative observations on habitat condition. As no fisheries statistics were available for $O$. nigrimas and other endemic fishes in Poso Lake, qualitative data on $O$. nigrimas exploitation and trends in abundance were obtained
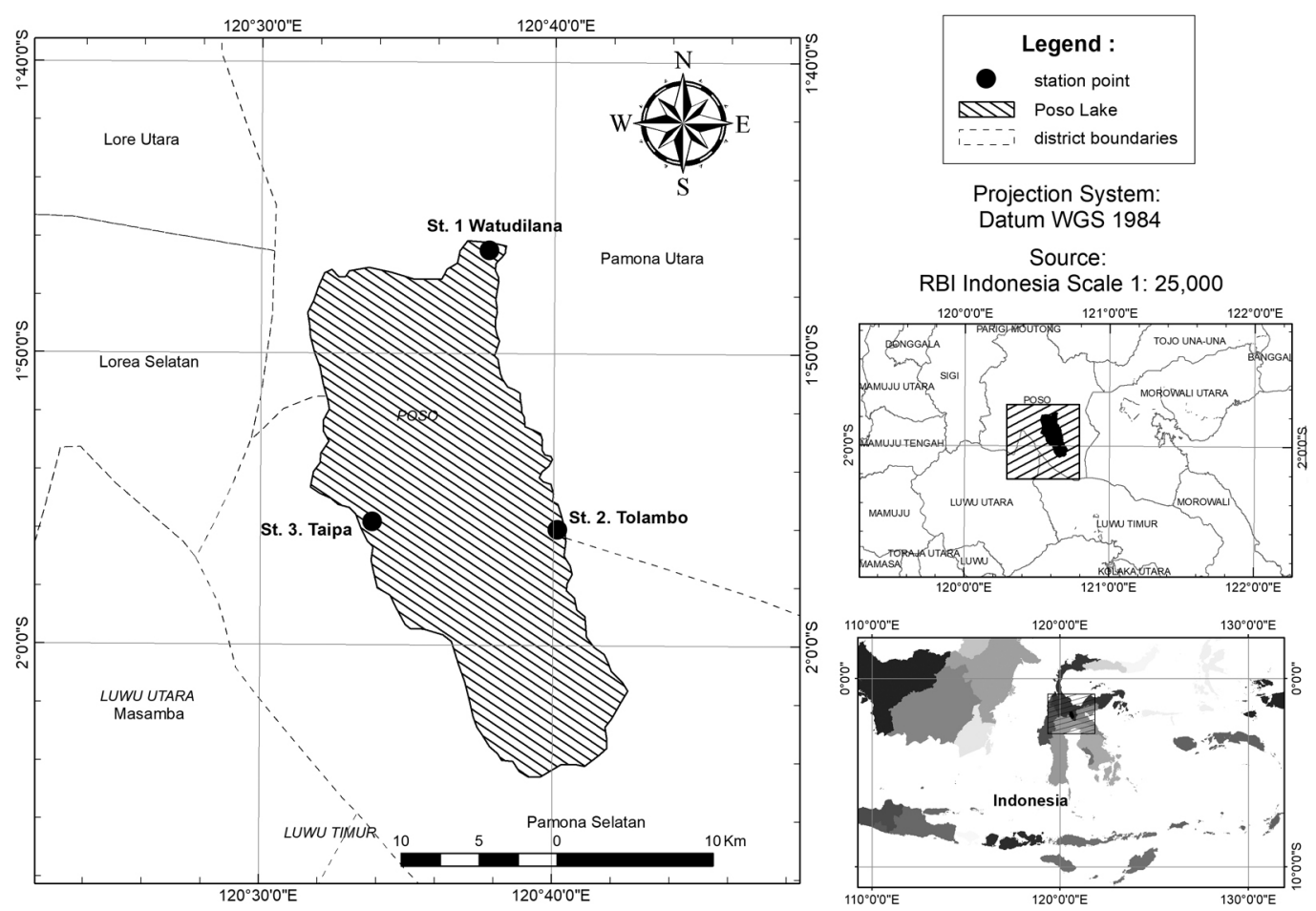

Fig. 1. Map of Poso Lake showing Oryzias nigrimas observation stations and the surrounding sub-districts (adapted from Serdiati et al., 2020). 
through direct observation and key informant interviews (government officials, fishermen, local residents). Secondary data were sourced from the scientific literature and databases, unpublished data (e.g. previous survey reports by academics), and other documents available online or from government agencies.

Data analysis: Data were analysed descriptively. A synopsis of bioecological parameters was compiled for of $O$. nigrimas and related species, focused on aspects relevant for conservation. Current and historical data on fish populations, habitat and exploitation levels were evaluated to identify actual or potential threats and changes relevant to the conservation of Poso endemic species, especially $O$. nigrimas. Management measures were formulated to reduce the risk of extinction and maintain or promote recovery of $O$. nigrimas populations along with other endemic species in Poso Lake.

\section{RESULTS}

Poso Lake ecosystem and $O$. nigrimas habitat: A synopsis of data (primary and secondary) on the habitat of O. nigrimas in Poso Lake is shown in Table 1. Table 2 presents an overview of major threats to the Poso Lake ecosystem in general and the habitat of $O$. nigrimas in particular.

Biology, ecology, conservation status and exploitation of $O$. nigrimas and other Poso ricefishes: A synopsis of data on key life history parameters for the black buntingi $O$. nigrimas (including data obtained during this study) and the other six Poso Lake endemic ricefishes (Table 3 ) reveals major gaps in current knowledge. Similar gaps are reflected in a synopsis of data on the ecology, conservation status and exploitation of Poso ricefishes (Table 4).

The fisheries targeting endemic fishes in Poso Lake were not regulated or recorded by any government agency. The field survey revealed that $O$. nigrimas and other Poso endemic ricefishes were among the species targeted by night-time fisheries using lights to attract phototaxis positive fish. The main light fishing gears were a variety of lift nets and gillnets; scoop nets were also used and beach seines also caught ricefishes. Lift nets generally operated 15-20 days per month, avoiding the full moon period. The $O$. nigrimas fishing grounds were in shallow waters close to the lake shore, and included all three survey sites (Fig. 1) where the rocky substrate

TABLE 1

Physical and chemical parameters of Poso Lake

\begin{tabular}{lccccccc}
\multicolumn{1}{c}{ Parameter (unit) } & \multicolumn{3}{c}{ Secondary data - Year } & \multicolumn{3}{c}{ This study (May 2017 to April 2018) - Station } \\
& $2007^{\mathrm{b}}$ & $2010^{\mathrm{c}}$ & $2011^{\mathrm{d}}$ & $2013^{\mathrm{e}}$ & 1 & 2 & 3 \\
Temperature $\left({ }^{\circ} \mathrm{C}\right)$ & $27.9-28.8^{\mathrm{f}}$ & 30.4 & $26-30$ & $27-28.5$ & $26.5-28$ & $26-28.5$ & $27.5-28.5$ \\
pH & $8.34-8.69$ & 7.7 & $6.39-8.09$ & $8.5-8.7$ & $8.0-8.5$ & $8.4-8.5$ & $8.3-8.5$ \\
DO (mg/l) & $5.91-7.80$ & 4.67 & $5.24-9.55$ & $5.86-5.99$ & $6.0-6.9$ & $6.0-6.8$ & $6.4-6.7$ \\
Visibility (m) & $10-11$ & na & $5.5-9$ & $7-11$ & $7.2-8.8$ & $8.5-10.6$ & $8.5-11.2$ \\
Nitrogen $(\mathrm{mg} / \mathrm{l})$ & $0.14-0.68$ & 0.42 & $0.07-0.15$ & na & na & na & na \\
Phosphate $(\mathrm{mg} / \mathrm{l})$ & $0.01-0.05$ & na & $0.04-0.05$ & na & na & na & na \\
Chlorophyll $\left(\mathrm{mg} / \mathrm{m}^{3}\right)$ & $0.24-1.69$ & na & na & na & na & na & na \\
N/P ratio & $2.5-39$ & na & na & na & na & na \\
Trophic status & mesotrophic to mildly eutrophic & oligotrophic & indications of eutrophication \\
Hardness & soft & na & na & na & na & na & na \\
Sedimentation & high & high & na & high & \multicolumn{7}{c}{ generally high } \\
\hline
\end{tabular}

${ }^{\mathrm{a}}$ na $=$ data not available; ${ }^{\mathrm{b}}$ Lukman (2007); ${ }^{\mathrm{c}}$ Mamondol (2018); ${ }^{\mathrm{d}}$ Makmur et al. (2011); ${ }^{\mathrm{e}}$ Gundo (2015); ${ }^{\mathrm{f}}$ mean value from $0-50 \mathrm{~m}$; temperature $\approx 27^{\circ} \mathrm{C}$ at $60 \mathrm{~m}$ and $29-29.5^{\circ} \mathrm{C}$ from $0-10 \mathrm{~m}$ depth. 
TABLE 2

Major threats to the Poso Lake ecosystem, including Oryzias nigrimas habitat

\begin{tabular}{|c|c|c|c|}
\hline Type of threat & Details & $\begin{array}{c}\text { Actual/potential impact on } O . \\
\text { nigrimas }\end{array}$ & References \\
\hline $\begin{array}{l}\text { Sedimentation, reduced } \\
\text { depth }\end{array}$ & $\begin{array}{l}\text { Erosion due to degradation } \\
\text { of the watershed, particularly } \\
\text { deforestation }\end{array}$ & $\begin{array}{l}\text { Degradation or loss of shallow- } \\
\text { water habitat, including hard } \\
\text { substrate and aquatic plants }\end{array}$ & $\begin{array}{l}\text { This study and } \\
\text { a,b,c,d,e,f }\end{array}$ \\
\hline $\begin{array}{l}\text { Lakeside development } \\
\text { including reclamation }\end{array}$ & $\begin{array}{l}\text { Growth of Tentena (the main } \\
\text { lakeside town) and other } \\
\text { settlements; tourism }\end{array}$ & $\begin{array}{l}\text { Pollution (sewage, run-off, plastic } \\
\text { and other garbage) and loss of } \\
\text { habitat, especially ecologically } \\
\text { important seasonally flooded } \\
\text { riparian areas }\end{array}$ & $\begin{array}{l}\text { This study and } \\
\mathrm{a,b,c,d,e,f,g}\end{array}$ \\
\hline $\begin{array}{l}\text { Aquaculture of non- } \\
\text { native species }\end{array}$ & $\begin{array}{l}\text { Mostly in net cages (fixed or } \\
\text { floating) in the Lake; some in Poso } \\
\text { River and tributaries }\end{array}$ & $\begin{array}{l}\text { Pollution, damage to habitat, and } \\
\text { accidental releases }\end{array}$ & This study and a,g,h,i,j \\
\hline $\begin{array}{l}\text { Introduced (alien) } \\
\text { invasive species }\end{array}$ & $\begin{array}{l}\text { Government projects and private } \\
\text { initiatives (species: see Table } 3 \text { ) }\end{array}$ & $\begin{array}{l}\text { Competition for habitat and food; } \\
\text { predation; degradation of habitat; } \\
\text { parasites and disease }\end{array}$ & This study and a,b,h,k \\
\hline $\begin{array}{l}\text { Spread of modern } \\
\text { farming and plantations }\end{array}$ & $\begin{array}{l}\text { Use of fertilisers and agricultural } \\
\text { chemicals; increased erosion and } \\
\text { changes in hydrology }\end{array}$ & $\begin{array}{l}\text { Eutrophication and other pollution } \\
\text { (e.g. pesticides); intensification of } \\
\text { floods, droughts and sedimentation } \\
\text { affecting water quality and water } \\
\text { level patterns }\end{array}$ & $\begin{array}{l}\text { This study and } \\
\text { a,b,c,d,e,f,k }\end{array}$ \\
\hline $\begin{array}{l}\text { Poso dam and } \\
\text { hydroelectric power } \\
\text { plants }\end{array}$ & $\begin{array}{l}\text { Effect on water level/ water flow } \\
\text { patterns and retention of sediments }\end{array}$ & $\begin{array}{l}\text { Potential impacts on seasonal } \\
\text { conditions; exacerbation of } \\
\text { problems related to sedimentation }\end{array}$ & This study and a,b,h \\
\hline
\end{tabular}

${ }^{\mathrm{a}}$ Gundo (2015); ${ }^{\mathrm{b}}$ IUCN Red List; ${ }^{\mathrm{c}}$ Nursangaji et al. (2014); ${ }^{\mathrm{d}}$ Lukman \& Ridwansyah (2009); ${ }^{\mathrm{e}}$ Mamondol (2018); ${ }^{\mathrm{f}}$ Nontji (2016); ${ }^{\mathrm{g}}$ Gundo (2010); ${ }^{\mathrm{h}}$ Herder et al. (2012); ${ }^{\mathrm{i}}$ Mokodongan (2019n); ${ }^{\mathrm{j}}$ Mokodongan (2019h); ${ }^{\mathrm{k}}$ Parenti \& Soeroto (2004).

is particularly suitable as $O$. nigrimas habitat. Fishermen and other locals consistently reported a decline in ricefish abundance with a decreasing trend in fisheries catch despite an increase in effort, indicating overfishing of O. nigrimas populations.

The mesh size of all liftnets and some gillnets observed was very small, making them intrinsically non-selective (size and species). The ricefish $O$. nebulosus was visually identified in the catch of fishers targeting O. nigrimas. This was confirmed by molecular (DNA) analysis (Serdiati et al., 2019b). Fishing activities targeting $O$. nigrimas were intensive and ricefishes in all age-size classes were caught. Very few captured ricefishes were in a fit condition to survive if certain categories (e.g. juvenile fish, egg-bearing females or specific species) were released.
Invasive alien species in Poso Lake: A growing number of non-native (alien invasive) species have been reported in Poso Lake. The list of introduced species reported in Poso Lake in Table 5 is likely incomplete.

The field survey revealed that local communities do not like eating these introduced fishes; with little fishing pressure, they have become established and spread. While the origins of some alien species (Table 5) are known (e.g. releases under government projects), others are speculative. The bony-lipped bard (Osteochilus vittatus) and Java barb (Barbonymus gonionotus), called nilem and tawes locally, are thought to have been released by migrants under the government "transmigrasi" program to reduce over-population in Java (and Bali). Nilem were the most abundant fish visible during a survey on Poso anguillid eels (Ndobe 
TABLE 3

Life history parameters of Poso Lake ricefishes ${ }^{\mathrm{a}}$

\begin{tabular}{|c|c|c|c|c|c|c|c|}
\hline Parameter (unit) & $\begin{array}{l}\text { Oryzias } \\
\text { nigrimas }\end{array}$ & $\begin{array}{c}\text { O. } \\
\text { orthognathus }\end{array}$ & $\begin{array}{c}\text { O. } \\
\text { nebulosus }\end{array}$ & $\begin{array}{c}\text { Xenopoecilus } \\
\text { oophorus }\end{array}$ & $\begin{array}{c}X . \\
\text { poptae }\end{array}$ & $\begin{array}{l}\text { Adrianichthys } \\
\text { kruyti }\end{array}$ & $\begin{array}{l}A . \\
\text { roseni }\end{array}$ \\
\hline $\begin{array}{l}\text { Main source (s) other } \\
\text { than FishBase }\end{array}$ & This study, ${ }^{\mathrm{c}}$ & d & This study, ${ }^{e, f}$ & $\mathrm{~g}, \mathrm{~h}$ & $\mathrm{i}, \mathrm{j}$ & $\mathrm{f}, \mathrm{k}$ & $\mathrm{f}, \mathrm{j}, 1$ \\
\hline $\begin{array}{l}\text { Maximum length } \mathrm{L}_{\max } \\
(\mathrm{mm}, \mathrm{TL} \text { or } \mathrm{SL})^{\mathrm{m}}\end{array}$ & $58.5 T L^{c} 52.5 S L^{c}$ & 65TL 50.1SL & $33 \mathrm{SL}$ & $\begin{array}{l}85 \mathrm{TL} \\
69.3 \mathrm{SL}\end{array}$ & $\begin{array}{l}171 \mathrm{SL} \\
192 \mathrm{SL}\end{array}$ & $\begin{array}{l}160 \mathrm{TL} \\
109 \mathrm{SL}\end{array}$ & 90SL \\
\hline $\begin{array}{l}\text { Length at first } \\
\text { maturity } \mathrm{L}_{\mathrm{m}}(\mathrm{mm})\end{array}$ & $50.8 \mathrm{M} ; 47.5 \mathrm{~F}^{\mathrm{c}}$ & na & na & na & na & na & na \\
\hline Estimated longevity & na & na & na & na & na & na & na \\
\hline Fecundity (eggs) & $143-243^{c}$ & na & na & $33-135$ & na & na & na \\
\hline Spawning pattern & partial $^{\mathrm{c}}$ & partial & na & partial & na & na & na \\
\hline $\begin{array}{l}\text { Spawning season } \\
\text { (month, abbreviated) }\end{array}$ & $\begin{array}{l}\text { Jun, Sept, Nov, } \\
\text { Dec, Feb }{ }^{c}\end{array}$ & na & na & $\begin{array}{l}\text { Nov, Jan, } \\
\text { Feb, Apr }\end{array}$ & na & na & na \\
\hline Sexual dimorphism & yes & yes & yes & yes & na & na & na \\
\hline $\begin{array}{l}\text { Parental care: external } \\
\text { (pelvic brooder) }\end{array}$ & $\begin{array}{c}\text { none (eggs laid } \\
\text { on vegetation) }\end{array}$ & na & $\begin{array}{c}\text { none (eggs } \\
\text { laid on vegetation) }\end{array}$ & yes & yes & yes & na \\
\hline Growth pattern & allometric negative $\mathrm{e}^{\mathrm{c}}$ & na & na & isometric & na & na & na \\
\hline Phototaxis $^{\mathrm{n}}$ & Positive & Positive & Positive & Positive & Positive & Positive & Positive \\
\hline
\end{tabular}

${ }^{\mathrm{a}}$ na $=$ data not available; bold characters indicate data from this study; ${ }^{\mathrm{b}}$ Froese \& Pauly (2020); ${ }^{\mathrm{c}}$ Serdiati (2019); ${ }^{\mathrm{d}}$ Mokodongan (2019h); ${ }^{\mathrm{e}}$ Mokodongan (2019g); ${ }^{\mathrm{f}}$ Parenti \& Soeroto (2004); ${ }^{\mathrm{g}}$ Gundo, Rahardjo, Batu, \& Hadie (2016); $\mathrm{h}$ Mokodongan (2019m); ${ }^{\mathrm{i}}$ Mokodongan (2019c); ${ }^{\mathrm{j}}$ Parenti (2008); ${ }^{\mathrm{k}}$ Mokodongan (2019p); ${ }^{1}$ Mokodongan $(2019 \mathrm{q}) ;{ }^{\mathrm{m} T L}=$ total length; $\mathrm{SL}=$ standard length; ${ }^{\mathrm{n}}$ From field data on light fishing.

TABLE 4

Ecology, conservation status and exploitation of Poso Lake endemic ricefishes ${ }^{\mathrm{a}}$

\begin{tabular}{|c|c|c|c|c|c|c|c|}
\hline Parameter/Aspect & $\begin{array}{c}\text { Oryzias } \\
\text { nigrimas }\end{array}$ & $\begin{array}{c}\text { O. } \\
\text { orthognathus }\end{array}$ & $\begin{array}{c}\text { O. } \\
\text { nebulosus }\end{array}$ & $\begin{array}{c}\text { Xenopoecilus } \\
\text { oophorus }\end{array}$ & X. poptae & $\begin{array}{c}\text { Adrianichthys } \\
\text { kruyti }\end{array}$ & A, roseni \\
\hline $\begin{array}{l}\text { Main source (s) } \\
\text { other than b,c }\end{array}$ & This study, ${ }^{d}$ & $\mathrm{e}$ & $f, g$ & $\mathrm{~g}$ & $\mathrm{e}$ & $\mathrm{e}$ & $\mathrm{e}$ \\
\hline Prior Red List status & VU & $\mathrm{EN}$ & none & EN & $\mathrm{CR}$ & $\mathrm{CR}$ & none \\
\hline 2019 Red List status & NT & NT & NT & $\mathrm{LC}$ & EN & $\mathrm{CR}$ & $\mathrm{CR}$ \\
\hline Population status ${ }^{\mathrm{h}}$ & $\mathrm{S}^{\mathrm{c}}$ or $\mathrm{D}$ & $\mathrm{S}$ & $\mathrm{S}$ & $\mathrm{S}$ & $\mathrm{U}$ & $\mathrm{U} / \mathrm{EX}$ & $\mathrm{U} / \mathrm{EX}$ \\
\hline $\begin{array}{l}\text { Pelagic (PL) or } \\
\text { Benthopelagic (BP) }\end{array}$ & PL & $\mathrm{BP} / \mathrm{PL}$ & PL & PL & PL & PL & na \\
\hline Feeding guild & omnivore ${ }^{\mathrm{d}, \mathrm{i}}$ & na & na & na & na & na & na \\
\hline $\begin{array}{l}\text { Areas in Poso Lake } \\
\text { (cardinal points) }\end{array}$ & W NE SEj & E W & E W & N E W & na & na & na \\
\hline Main threats ${ }^{\mathrm{k}}$ & $\mathrm{IN}, \mathrm{HL}, \mathrm{IF}^{\mathrm{f}}$ & IN, HL & IN, HL, IF, $\mathrm{OT}^{\mathrm{f}}$ & IN, HL, IF & IN, HL & IN & IN \\
\hline $\begin{array}{l}\text { Known to be fished - } \\
\text { food fish }\end{array}$ & yes & yes $^{1}$ & $\operatorname{yes}^{f}$ & yes $^{\mathrm{m}}$ & yes $^{n}$ & yes $^{0}$ & yes $^{f}$ \\
\hline Aquarium trade & yes $^{b}$ & yes $^{b}$ & proposed $^{\mathrm{f}}$ & proposed $^{\mathrm{f}}$ & na & na & na \\
\hline
\end{tabular}

${ }^{\mathrm{a}}$ na = data not available; bold characters indicate data from this study; ${ }^{\mathrm{b}}$ Froese \& Pauly $(2020) ;{ }^{\mathrm{c}}$ The IUCN Red List; ${ }^{\mathrm{d}}$ Serdiati et al. (2019a); ${ }^{\mathrm{e}}$ Parenti (2008); ${ }^{\mathrm{f}}$ Parenti \& Soeroto (2004); ${ }^{\mathrm{g}}$ Gundo (2015); ${ }^{\mathrm{h}} \mathrm{S}=$ stable; I = increasing; D = declining, $\mathrm{U}=$ unknown; U/EX: unknown, possibly extinct; ${ }^{\mathrm{i}}$ Predominantly herbivorous; ${ }^{\mathrm{j}}$ This study: all three survey stations (W, NE and SE; Fig. 1): Mokodongan (2019n): only West (W); ${ }^{\mathrm{k}} \mathrm{IN}=$ invasive species; $\mathrm{HL}=$ habitat loss/degradation; IF = intensive fishing; OT = other (infestation with parasitic copepods, Lernaea sp.); ${ }^{1}$ Mokodongan $(2019 \mathrm{~h}) ;{ }^{\mathrm{m}}$ Mokodongan $(2019 \mathrm{~m}) ;{ }^{\mathrm{n}}$ Mokodongan (2019c); ${ }^{\circ}$ Mokodongan (2019p). 
TABLE 5

Non-native species reported in Poso Lake

\begin{tabular}{|c|c|c|c|c|c|}
\hline No & Common name & Scientific name & Local name & Remarks $^{\text {a }}$ & Reference \\
\hline 1 & Nile tilapia & Oreochromis niloticus & Ikan nila & INV, PR, CP, HB, FF, G & $\mathrm{d}, \mathrm{e}, \mathrm{f}, \mathrm{i}, \mathrm{j}$ \\
\hline 2 & Tilapia & O. mossambicus & Ikan mujair & INV, PR, CP, HB, FF, G & $\mathrm{d}, \mathrm{e}, \mathrm{j}$ \\
\hline 3 & Common carp & Cyprinus carpio & Ikan mas & INV, CP, HB, FF, G & $\mathrm{d}, \mathrm{f}, \mathrm{j}$ \\
\hline 4 & Bonylip barb & Osteochilus vittatus $^{b}$ & Ikan nilem & INV, CP & $\mathrm{d}, \mathrm{e}, \mathrm{f}, \mathrm{i}$ \\
\hline 5 & Java barb & Barbonymus gonionotus & Ikan tawes & INV, CP & $\mathrm{d}, \mathrm{e}, \mathrm{f}$ \\
\hline 6 & Catfish & Clarias sp. & Ikan lele & INV, PR, HB, FF, G & $\mathrm{d}, \mathrm{f}, \mathrm{h}, \mathrm{j}$ \\
\hline 7 & Pangasius & Pangasius sp. & Ikan patin & INV, PR, HB, FF, G & $\mathrm{e}$ \\
\hline 8 & Tambaqui, $\mathrm{pacu}^{\mathrm{k}}$ & Colossoma macropoтum & Bawal air tawar & INV, PR, HB, FF, G & $\mathrm{d}, \mathrm{e}$ \\
\hline 9 & Flowerhorn cichlid & none: hybrid cichlid & Flowerhorn & INV, PR, HB, FF, U A & $\mathrm{e}$ \\
\hline 10 & Auratus cichlid & Melanochromis auratus & Cichlid Afrika & INV, PR, HB, FF, U & $\mathrm{e}$ \\
\hline 11 & Three-spot gourami & Trichopodus trichopterus & Ikan sepat & $\begin{array}{l}\mathrm{INV}, \mathrm{PR}, \mathrm{HB}, \mathrm{FF}, \mathrm{U} \\
\text { Possibly also } T \text {. pectoralis }\end{array}$ & e,f, \\
\hline 12 & Blue panchax & Aplocheilus panchax & Ikan kepala timah & $\mathrm{INV}, \mathrm{CP}, \mathrm{U}$ & $\mathrm{e}$ \\
\hline 13 & Bichir & Polypterus sp. & Ikan palmas & African origin & d \\
\hline 14 & Guppy & Poecilia reticulata & Ikan seribu & INV, CP, U & $\mathrm{e}$ \\
\hline 15 & Swordtail & Xiphophorus helleri & Ikan pedang & INV, U, IU & d \\
\hline 16 & Platyfish & Xiphophorus maculatus & Ikan platy & INV, U, IU & d \\
\hline 17 & Janitor fish & Family Locariidae $^{c}$ & Ikan sapu-sapu & INV, PR, HB, U & $\mathrm{g}$ \\
\hline 18 & Climbing perch & Anabas testudineus & Ikan betok & $\mathrm{PN}, \mathrm{PR}$ & $\mathrm{e}, \mathrm{f}, \mathrm{h}, \mathrm{i}$ \\
\hline 19 & Striped snakehead & Channa striata & Ikan gabus & PN, PR & $\mathrm{e}, \mathrm{f}, \mathrm{h}, \mathrm{i}$ \\
\hline
\end{tabular}

${ }^{\mathrm{a}} \mathrm{INV}=$ invasive; $\mathrm{PN}=$ possibly native; $\mathrm{PR}=$ known or suspected predator; $\mathrm{CP}=$ known or suspected competitor for resources (e.g. food, habitat); $\mathrm{HB}=\mathrm{known} /$ suspected to cause habitat degradation; $\mathrm{FF}=$ food fish (caught by local fishers); $\mathrm{G}=$ introduced under government projects; $\mathrm{U}=$ unknown origin; $\mathrm{IU}=$ impact unknown; ${ }^{\mathrm{b}}$ Synonym $O$. hasselti; ${ }^{\mathrm{c}}$ Taxonomy of this family is confused; most likely Hypostomus sp. or Pterygoplichthys sp.; ${ }^{\mathrm{d}}$ Parenti \& Soeroto (2004); ${ }^{\mathrm{e}}$ Gundo (2015); ${ }^{\mathrm{f}}$ Whitten, Henderson, \& Mustafa (2002); ${ }^{\mathrm{g}}$ Gundo pers. com (2018); ${ }^{\mathrm{h}}$ Herder et al. (2012); ${ }^{\mathrm{I}}$ Makmur et al. (2011); ${ }^{\mathrm{j}}$ IUCN Red List; ${ }^{k}$ may include other species of the genera Colossoma and Piaractus.

and Moore, unpublished data, 2008), in an area where endemic (native) species were abundant during surveys a decade earlier (Ndobe, unpublished data, 1996-2001). Several species seem to have been introduced through escape from aquaculture facilities or through well-meaning but misguided release to the wild of ornamental fishes (Parenti \& Soeroto, 2004; Samliok Ndobe \& Abigail Moore, unpublished data, 2008). One of the more recent and potentially most dangerous introductions for endemic fish species, the janitor fish (ikan sapu-sapu), Family Locariidae, was documented in Poso Lake in 2018 (Gundo, pers. com., 2018).

Management options for $O$. nigrimas. Based on the data presented above, proposed priorities, interventions and anticipated benefits are outlined in Table 6. One potential intervention is the development of captive breeding followed by restocking. The national guidelines for restocking threatened aquatic (marine and freshwater) species (Sadili, Haryono, Kamal, Sarmintohadi, \& Ramli, 2015 ) and their relevance to $O$. nigrimas are summarised in Table 7.

\section{DISCUSSION}

Need for a holistic approach: The direct and indirect threats to Oryzias nigrimas (e.g. habitat degradation and loss, introduced alien species, and intensive exploitation as a locally important food fish) are complex and 
TABLE 6

Matrix of proposed $O$. nigrimas conservation priorities and interventions

\begin{tabular}{|c|c|c|c|}
\hline No & Priority & Interventions & Anticipated benefits/risks \\
\hline 1 & $\begin{array}{l}\text { Data for } \\
\text { management }\end{array}$ & $\begin{array}{l}\text { Design and implement a comprehensive } \\
\text { baseline survey and monitoring program for } \\
\text { the ichthyofauna of Poso Lake, with focus on } \\
\text { endemic species }\end{array}$ & $\begin{array}{l}\text { Spatial distribution of species and habitat use } \\
\text { Basis for stock assessments and fisheries } \\
\text { management as well as conservation planning } \\
\text { Basis for evaluation of management success }\end{array}$ \\
\hline 2 & $\begin{array}{l}\text { Reduce direct } \\
\text { threats (fishery- } \\
\text { related) }\end{array}$ & $\begin{array}{l}\text { Immediate: spatial and temporal restrictions } \\
\text { on fishing/gear use based on existing data } \\
\text { Revisions based on outputs from point } 1\end{array}$ & $\begin{array}{l}\text { Increased survival and reproductive potential of } \\
\text { O. nigrimas/ other endemic fishes } \\
\text { Potential economic impact: may need research } \\
\text { on selective gear to reduce endemic species } \\
\text { by-catch }\end{array}$ \\
\hline 3 & $\begin{array}{l}\text { Mitigate alien } \\
\text { species impacts }\end{array}$ & $\begin{array}{l}\text { Strict regulations on release of aquatic } \\
\text { organisms in Poso Lake and watershed, } \\
\text { with effective surveillance and enforcement } \\
\text { (penalties) } \\
\text { Cessation of so-called "re-stocking" of alien } \\
\text { species } \\
\text { Encourage exploitation of introduced species } \\
\text { Control of alien species not used as food } \\
\text { fish (e.g. sapu-sapu), including research on } \\
\text { possible uses to encourage fishing }\end{array}$ & $\begin{array}{l}\text { Increased survival and reproductive success of } \\
\text { endemic fishes, including } O \text {. nigrimas } \\
\text { Eradication of feral alien species unlikely } \\
\text { to succeed, so control measures will need to } \\
\text { be continuous and long-term; need to seek } \\
\text { profitable ways to control so that fishers will do } \\
\text { the work as part of their livelihoods } \\
\text { Need for research to ensure control measures do } \\
\text { not impact endemic fish/invertebrates }\end{array}$ \\
\hline 4 & $\begin{array}{l}\text { Reduce indirect } \\
\text { threats }\end{array}$ & $\begin{array}{l}\text { Integrated watershed management: synergy } \\
\text { with Lake Rehabilitation Program (Nursangaji } \\
\text { et al., 2014) and relevant agencies/stakeholder } \\
\text { groups }\end{array}$ & $\begin{array}{l}\text { Prevent (or at least minimise) decline in habitat } \\
\text { condition, especially water quality and impacts } \\
\text { of sedimentation } \\
\text { Potential for habitat rehabilitation }\end{array}$ \\
\hline 5 & $\begin{array}{l}\text { Ex-situ research } \\
\text { and breeding }\end{array}$ & $\begin{array}{l}\text { In-depth research on } O \text {. nigrimas reproductive } \\
\text { biology/feeding habits o } \\
\text { Develop breeding and release protocols, with } \\
\text { due attention to guidelines (Table } 7 \text { ) }\end{array}$ & $\begin{array}{l}\text { Improved understanding to inform in-situ } \\
\text { management } \\
\text { Captive population as genetic bank and potential } \\
\text { source of fish for eventual restocking } \\
\text { Potential as additional «model organism» }\end{array}$ \\
\hline
\end{tabular}

interrelated with each other as well as with the status of other species (native and introduced) in Poso Lake. Ensuring the survival of this species in its native (endemic) habitat therefore requires a holistic approach, crossing sociopolitical boundaries (e.g. between government sectors, scientific disciplines, administrative jurisdictions and community segments/actors), as well as linking aquatic and terrestrial ecosystems. Action to mitigate these threats should benefit other endemic fish and invertebrate species in Poso Lake. Proposed approaches discussed below under four thematic groupings would each benefit greatly from and should be synergised with the other approaches.

Habitat conservation and rehabilitation: Like other ancient lakes, Poso Lake was until quite recently classified as oligotrophic (Giesen, 1994). Various human activities around the lake and throughout the watershed have increased both nutrient levels and sedimentation rates in the lake (Nursangaji et al., 2014; Nontji, 2016; Mamondol, 2018). Increased nutrients are thought to arise from urbanisation around the Lake as well as the increased use of fertiliser on arable and plantation crops which have increasingly replaced the natural forest vegetation and waste from cage culture of introduced fishes. The loss of around 50-60 $\mathrm{m}$ in maximum depth over four decades (Lukman \& Ridwansyah, 2009) is most likely related to increasing rates of erosion in the Poso Lake watershed (Lukman \& Ridwansyah, 2009; Nursangaji et al., 2014; Mamondol, 2018). Erosion rates of 15-60 tonnes/ha/year 
TABLE 7

Summary of key considerations in the Indonesian national guidelines for restocking threatened fishes (Sadili et al., 2015)

\begin{tabular}{|c|c|c|}
\hline No & Guideline component & Relevance to $O$. nigrimas \\
\hline 1 & $\begin{array}{l}\text { Where to restock: water bodies (marine or freshwater) } \\
\text { where threatened species occur naturally and native } \\
\text { species populations have declined severely }\end{array}$ & $\begin{array}{l}\text { Poso Lake qualifies - for the native (especially endemic) } \\
\text { species in the lake }\end{array}$ \\
\hline 2 & $\begin{array}{l}\text { What to restock: native species, within parts of their native } \\
\text { range where they have been extirpated or populations have } \\
\text { declined to a level unlikely to permit natural recovery. In } \\
\text { the case of exploited species, restocking might also be } \\
\text { appropriate as part of a sustainable fisheries management } \\
\text { strategy }\end{array}$ & $\begin{array}{l}\text { O. nigrimas qualifies as a threatened species, despite the } \\
\text { edowngrading of Red List category in } 2019 \text { (Mokodongan } \\
2019 \text { n); however, with the population assessed as «stable» } \\
\text { it does not qualify for restocking at present }\end{array}$ \\
\hline 3 & $\begin{array}{l}\text { When to restock: once the factors which caused } \\
\text { extirpation/drastic decline have been eliminated or } \\
\text { mitigated and measures are in place to promote survival/ } \\
\text { sustainable management }\end{array}$ & $\begin{array}{l}\text { These aspects have not yet been addressed for } O \text {. } \\
\text { nigrimas, but should include attention to habitat and } \\
\text { threats from introduced species. }\end{array}$ \\
\hline 4 & $\begin{array}{l}\text { Fish used for restocking: progeny of native fish, } \\
\text { representing maximum possible genetic variation in native } \\
\text { population; in good health (free of pests and diseases); } \\
\text { adapted for release and survival in the wild (e.g. not } \\
\text { acclimated to pelleted feed) }\end{array}$ & $\begin{array}{l}\text { The necessary knowledge, technology and protocols for } \\
\text { breeding and release would need to be developed for } O \text {. } \\
\text { nigrimas, through fundamental and applied research. }\end{array}$ \\
\hline 5 & $\begin{array}{l}\text { Post-restocking: monitoring and evaluation are vital (and } \\
\text { require valid initial baseline data) }\end{array}$ & Baseline not yet available. Monitoring not yet in place. \\
\hline
\end{tabular}

have been reported in the watershed around Poso Lake, with the highest rates in areas with predominantly steep slopes and high rates of deforestation (Mamondol, 2018). The data in Table 1 and Table 2 indicate an ongoing degradation of the Poso Lake ecosystem in general and $O$. nigrimas habitat in particular.

On the positive side, there are initiatives with potential to mitigate or even to some extent reverse environmental degradation, as highlighted in Table 6. In particular, as Poso Lake is one of 15 lakes in the national lake rehabilitation program (Nursangaji et al., 2014), there are many opportunities for synergy with this program. A key recommendation is that this and other Poso Lake watershed programs should include endemic species as a priority factor. Specific measures for endemic fish conservation should be synergised with this program, including direct protection of key habitat and habitat restoration through improved watershed management and restoration.
Sustainable ricefish fisheries in Lake Poso: Data collection for fisheries purposes (Poso Lake and surrounding watershed) has concentrated on anguillid eels and introduced food fish species (e.g. Makmur et al., 2011). The 2019 IUCN Red List assessments referring to surveys in 2012 and 2017 (Mokodongan, 2019c, 2019g, 2019h, 2019m, 2019n, 2019p, 2019q) and research on O. nigrimas and Xenopoecilus oophorus (Gundo, 2010; Gundo, 2015) confirm the field survey findings that Poso endemic ricefishes in general are target and/or bycatch species in light fisheries which are of importance for local food security and livelihoods.

The biology and ecology of most Sulawesi freshwater endemic species is still poorly understood, including the Poso Lake ricefishes in general and O. oryzias in particular (Table 3, Table 4). Knowledge gaps include ecological roles and interactions in the environments in which they have evolved as well as parameters 
used in both traditional and ecosystem-based approaches to fisheries management. Despite these gaps, we recommend an assessment of the Poso ricefish fisheries using the Ecosystem Approach to Fisheries Management (EAFM) indicators developed primarily for marine fisheries in Indonesia (NWG-EAFM, 2014; Pomeroy et al., 2015) and currently being adapted to freshwater fisheries.

As reported previously for the $X$. oophorus fishery (Gundo, 2010; Gundo, 2015), the intensive and currently unregulated light-assisted liftnets arguably pose the most serious direct fishing-related threat to Poso Lake endemic fishes, including $O$. nigrimas. By their very nature, these liftnets are unselective in terms of species and size. Therefore, attempts to reduce or manage the impact of these gears on endemic fish populations need to focus on temporal and spatial aspects. Specific priorities include spatial (key feeding, spawning and nursery grounds) and temporal (peak spawning periods) protection for $O$. nigrimas populations. The current de facto temporal fishing limitation to darker nights, during which light fishing is most effective, could be adapted to conserve fisheries resources, in particular the phototaxis positive endemic ricefish populations in Poso Lake. November and February appear to be key spawning seasons for both $O$. nigrimas and $X$. oophorus (Table 3 ). Extending the non-fishing period to the spawning peaks of the ricefishes present at each fishing site should increase the number of fish reproducing before capture, and thus help to maintain stocks. Spatial restrictions could include fisheries reserves (suaka perikanan in Indonesia), to support the conservation (including sustainable use) of $O$. nigrimas and other endemic fishes in Poso Lake.

While data poor approaches could be adopted now, we recommend multidisciplinary studies to fill the many knowledge gaps, in particular to identify spawning periods, investigate trophic ecology and map key habitats of Poso Lake endemic species, as well as to collect data on preferred fishing grounds and monitor catch compositions. Such data would enable the identification of times and places where temporal and/or spatial restrictions could have a maximum positive impact on endemic fish resources while minimising negative impacts on subsistence and commercial fisheries.

A routine monitoring program for $O$. nigrimas and other Poso Lake endemic species is needed to build time series data sets as a basis for science-based adaptive and holistic management. Sustainable levels and patterns of exploitation could support local livelihoods including fisheries and other economic activities (e.g. eco-tourism) and maintaining local culture, including culinary specialties based on $O$. nigrimas and other endemic fishes.

The conundrum of alien species: Among the threats to the endemic Ichthyofauna of Poso Lake, introduced (alien) species are a particular concern. Government projects, misleadingly called "restocking" programs, have (often repeatedly) introduced non-native species. Several of these are known to be invasive and have negatively impacted native ichthyofauna in Indonesia and other countries, including two species of Tilapia (Oreochromis niloticus and O. mossambicus); African catfish Clarias sp.; and aggressive and omnivorous South American piranha relatives of the genera Colossoma and Piaractus (Cagauan, 2007; Sadili et al., 2015; Ndobe et al., 2019).

The status of two species, the striped snakehead Channa striata (local name ikan gabus) and climbing perch Anabas testudineus (local name ikan betok), is unclear. Some regard these species as native to (at least parts) of Sulawesi (Kottelat, Whitten, Kartikasari, \& Wirjoatmodjo, 1996) while others consider them introduced (Herder et al., 2012). Both are considered at risk from introduced species in some areas of Central Sulawesi (Ndobe et al., 2019). If introduced by humans, their place in local culture and cuisine indicates the introductions must have occurred a long time ago, centuries or even millennia. Considering that all known Poso endemic fishes (including those considered critically endangered or possibly even extinct) have been found in recent 
decades, it is unlikely that $A$. testudineus or $C$. striata posed a significant threat to Poso ricefishes in the past. However, being predatory fishes, they could potentially accelerate the decline or impede recovery of ricefish populations severely depleted from other causes.

The national guidelines for restocking threatened aquatic (marine and freshwater) species (Sadili et al., 2015, summarised in Table 7) advocate precautionary approaches. It is unfortunate that similar precautions have rarely (if ever) been followed for the introduction of non-native fishes (by government and nongovernment parties) across Indonesia, most of which have occurred without any scientific evaluation of the risks to native biodiversity (including locally important fisheries species) and ecosystems. This paradigm is reflected in the list of introduced (alien invasive) species in Poso Lake (Table 5). It is vital to avoid further introductions (of existing or new alien species), and serious efforts should be made to mitigate the impacts of past alien species introductions.

No reports could be found of successful eradication after breeding populations were established of the invasive species already present in Poso Lake from any other natural lakes. It seems logical that fisheries for alien species should be encouraged to control (and hopefully reduce) their abundance and environmental impact. However, it is crucial that such fisheries are operated (and regulated) in ways which minimise negative impacts on $O$. nigrimas and other endemic species, both direct (e.g. as by-catch or through behavioural disturbance) and indirect (e.g. through impacts on habitat, such as lacustrine vegetation and water quality).

While eradication of introduced species is unlikely to be feasible on practical grounds (and in some cases livelihood or food security considerations), the prevention of further introductions should be a priority. Government should lead the way, ceasing so-called "restocking" programs which are in fact alien species introductions, and implementing mitigation measures. Education and public awareness strategies need to be accompanied by effective surveillance and enforcement, with meaningful penalties strictly applied for any violations such as the release of alien species.

Potential contribution of aquaculture and ex-situ measures: In addition to in-situ conservation measures, ex-situ research and conservation-oriented breeding could be considered for $O$. nigrimas and other endemic ricefishes. The medaka $O$. latipes is widely-used as a model organism, and ricefishes are considered relatively easy to maintain and breed in captivity (Parenti \& Soeroto, 2004; Hilgers \& Schwarzer, 2019). Several Sulawesi ricefishes have been successfully bred in the laboratory (Parenti \& Soeroto, 2004). Recent research on the food habits of $O$. nigrimas (Serdiati et al., 2019a) provides data for the development of captive breeding for this species.

Restocking is not considered necessary at present for $O$. nigrimas, based on the condition of the endemic population. Furthermore, the scientific/technical basis has not yet been developed and the necessary knowledge is not available regarding historical range of $O$. nigrimas in Poso Lake. However, research to develop the knowledge and tools to support an eventual need for responsible restocking is highly recommended, both as "insurance" and as a means of learning and building the scientific basis and human capacity to support in-situ management. True restocking (sensu Sadili et al., 2015) of native/endemic fish species should follow the guidelines in Table 7. The development of ex-situ breeding could also potentially support aquaculture as an economic activity, and reduce fisheries pressure.

\section{ACKNOWLEDGMENTS}

The authors acknowledge support from the Indonesian Ministry of Research, Technology and Higher Education of the Republic of Indonesia through a doctoral scholarship to the first author. The authors also wish to thank all who in any way contributed to the research, as well as the preparation and publication of this manuscript. In particular, we acknowledge 
the valuable and insightful contribution of the anonymous reviewers.

\section{RESUMEN}

Perspectivas sobre el manejo sostenible del pez Oryzias nigrimas, endémico del Lago Poso en Indonesia (Actinopterygii: Adrianichthyidae). Introducción: Los peces endémicos de los antiguos lagos de Sulawesi están cada vez más amenazados. Objetivo: Evaluar los datos y la información disponible desde una perspectiva de gestión holística y formular medidas para conservar el pez endémico Oryzias nigrimas en el lago Poso, Indonesia. Métodos: recopilación de datos primarios de tres estaciones alrededor del lago Poso y revisión de la literatura. Resultados: Las amenazas para $O$. nigrimas incluyen la degradación y pérdida del hábitat, la introducción de especies exóticas y la explotación como producto pesquero de importancia local. Las opciones para promover la ordenación pesquera sostenible incluyen limitaciones espaciales y temporales para minimizar la captura de peces grávidos o reproductores. La protección del hábitat debe incluir medidas para minimizar los impactos de las actividades que pueden reducir la calidad del agua y perturbar o matar la vegetación acuática. Conclusión: Las medidas para prevenir una disminución de la población de $O$. nigrimas se consideran urgentes y se recomienda realizar más investigaciones para llenar los vacíos de conocimiento identificados. La conservación ex situ, incluido el desarrollo de la cría en cautividad, también podría contribuir a una estrategia de conservación holística de $O$. nigrimas.

Palabras clave: endemismo; lacustre; Oryzae; medaka negro; especies invasivas; pesca con luces.

\section{REFERENCES}

Cagauan, A.A. (2007). Exotic aquatic species introduction in the Philippines for aquaculture-A threat to biodiversity or a boon to the economy? Journal of Environmental Science and Management, 10, 48-62.

Froese, R., \& Pauly, D. (2020). FishBase. The Global Database of Fishes WWW Document. Retrieved from http://www.fishbase.org

Giesen, W. (1994). Indonesia's major freshwater lakes: A review of current knowledge, development processes and threats. Internationale Vereinigung für Theoretische und Angewandte Limnologie: Mitteilungen, 24(1), 115-128.

Gundo, M.T. (2010). Daerah penangkapan dan status alat tangkap ikan rono (Xenopoecilus oophorus) di Danau Poso. Media Litbang Sulteng, 3, 33-37.

Gundo, M.T. (2015). Konservasi ikan endemik rono, Xenopoecillus oophorus, Kottelat 1990 di Danau Poso,
Sulawesi Tengah. Bogor, Indonesia: Bogor Agricultural University.

Gundo, M.T., Rahardjo, M.F., Batu, D.T.F.L., \& Hadie, W. (2016). Reproductive characteristics of female eggcarrying buntingi, Xenopoecilus oophorus, an endemic fish to Lake Poso in Central Sulawesi. Makara Journal of Science, 20(2), 88-94.

Hadiaty, R.K. (2018). Taxonomical status of endemic freshwater ichthyofauna of Sulawesi. Jurnal Iktiologi Indonesia, 18(2), 175-190.

Herder, F., Schliewen, U.K., Geiger, M.F., Hadiaty, R.K., Gray, S.M., McKinnon, J.S., ... Pfaender, J. (2012). Alien invasion in Wallace's dreamponds: Records of the hybridogenic "flowerhorn" cichlid in lake Matano, with an annotated checklist of fish species introduced to the Malili Lakes system in Sulawesi. Aquatic Invasions, 7(4), 521-535.

Hilgers, L., \& Schwarzer, J. (2019). The untapped potential of medaka and its wild relatives. Elife, 8, e46994.

Kottelat, M. (2013). The fishes of the inland waters of Southeast Asia: A catalogue and core bibliography of the fishes known to occur in freshwaters, mangroves and estuaries. The Raffles Bulletin of Zoology, Supplement 27, 1-663.

Kottelat, M., Whitten, A.J., Kartikasari, S., \& Wirjoatmodjo, S. (1996). Freshwater fishes of Western Indonesia and Sulawesi. Singapore, Singapore: Periplus TD.

Lukman, S. (2007). Karakteristik sejarah kehidupan ikan sidat (Anguilla sp.) di DAS Poso Sulawesi Tengah. Indonesia, Jakarta: Lembaga Ilmu Pengetahuan Indonesia.

Lukman, S., \& Ridwansyah, I. (2009). Telaah kondisi fisik Danau Poso dan prediksi ciri ekosistem perairannya. Limnotek, 26(2), 64-73.

Lumbantobing, D. (2019a). Oryzias celebensis. WWW Document IUCN Red List of Threatend Species 2019. Retrieved from http://dx.doi.org/10.2305/ IUCN.UK.2019-2.RLTS.T15579A90980558.en

Lumbantobing, D. (2019b). Oryzias hadiatyae. WWW Document IUCN Red List of Threatend Species 2019. Retrieved from http://dx.doi.org/10.2305/ IUCN.UK.2019- 2.RLTS.T90980607A90980632.en

Lumbantobing, D. (2019c). Oryzias profundicola. WWW Document IUCN Red List of Threatend Species 2019. Retrieved from http://dx.doi.org/10.2305/ IUCN.UK.2019-2.RLTS.T15578A90980796.en

Lumbantobing, D. (2019d). Oryzias marmoratus. WWW Document IUCN Red List of Threatend Species 2019. Retrieved from http://dx.doi.org/10.2305/ IUCN.UK.2019-2.RLTS.T15574A90980644.en

Makmur, S., Sawestri, S., Atminarso, D., Pongmasak, P.R., Selamet, S., Jahri, M., \& Irawan, B. (2011). 
Ekobiologi dan kegiatan perikanan di Perairan Danau Poso Sulawesi Tengah. Palembang, Indonesia: Kementerian Kelautan dan Perikanan.

Mamondol, M.R. (2018). Fungsi strategis Danau Poso, gangguan keseimbangan ekosistem, dan upaya penanggulangannya In Asosiasi Teolog Indonesia (Ed.), Prosiding Simposium Asosiasi Teolog Indonesia Regional Sulawesi, Tentena, Poso, Sulawesi Tengah (pp. 1-25). Jakarta, Indonesia: Asosiasi Teo$\log$ Indonesia.

Mandagi, I.F., Mokodongan, D.F., Tanaka, R., \& Yamahira, K. (2018). A new riverine ricefish of the genus Oryzias (Beloniformes, Adrianichthyidae) from Malili, Central Sulawesi, Indonesia. Copeia, 106, 297-304.

Meisner, A.D. (2001). Phylogenetic systematics of the viviparous halfbeak genera Dermogenys and Nomorhamphus (Teleostei : Hemiramphidae: Zenarchopterinae). Zoological Journal of the Linnean Society, 133, 199-283.

Meixner, M.J., Lüter, C., Eckert, C., Itskovich, V., Janussen, D., von Rintelen, T., ... Hess, W.R. (2007). Phylogenetic analysis of freshwater sponges provide evidence for endemism and radiation in ancient lakes. Molecular Phylogenetics and Evolution, 45(3), 875-886.

Mokodongan, D.F. (2019a). Oryzias javanicus. WWW Document IUCN Red List of Threatend Species 2019. Retrieved from http://dx.doi.org/10.2305/ IUCN.UK.2019- 2.RLTS.T89808701A89808742.en

Mokodongan, D.F. (2019b). Xenopoecilus bonneorum WWW Document IUCN Red List of Threatend Species 2019. Retrieved from http://dx.doi.org/10.2305/ IUCN.UK.2019- 2.RLTS.T90980978A90980999.en

Mokodongan, D.F. (2019c). Xenopoecilus poptae. WWW Document IUCN Red List of Threatend Species 2019. Retrieved from http://dx.doi.org/10.2305/ IUCN.UK.2019-2.RLTS.T23122A90980908.en

Mokodongan, D.F. (2019d). Xenopoecilus sarasinorum. WWW Document IUCN Red List of Threatend Species 2019. Retrieved from http://dx.doi.org/10.2305/ IUCN.UK.2019- 2.RLTS.T23123A90980952.en

Mokodongan, D.F. (2019e). Oryzias matanensis. WWW Document IUCN Red List of Threatend Species 2019. Retrieved from http://dx.doi.org/10.2305/ IUCN.UK.2019-2.RLTS.T15575A90980691.en

Mokodongan, D.F. (2019f). Oryzias asinua. WWW Document IUCN Red List of Threatend Species 2019. Retrieved from http://dx.doi.org/10.2305/IUCN. UK.2019-2.RLTS.T125851287A125851318.en

Mokodongan, D.F. (2019g). Oryzias nebulosus. WWW Document IUCN Red List of Threatend Species 2019. Retrieved from http://dx.doi.org/10.2305/ IUCN.UK.2019- 2.RLTS.T90980832A90980842.en
Mokodongan, D.F. (2019h). Oryzias orthognathus. WWW IUCN Red List of Threatend Species 2019. Retrieved from http://dx.doi.org/10.2305/IUCN.UK.2019-2. RLTS.T15577A90980762.en

Mokodongan, D.F. (2019i). Oryzias woworae. WWW Document IUCN Red List of Threatend Species 2019. Retrieved from http://dx.doi.org/10.2305/ IUCN.UK.2019- 2.RLTS.T90332191A90332196.en

Mokodongan, D.F. (2019j). Oryzias wolasi. WWW Document IUCN Red List of Threatend Species 2019. Retrieved from http://dx.doi.org/10.2305/IUCN. UK.2019- 2.RLTS.T125851303A125851309.en

Mokodongan, D.F. (2019k). Oryzias soerotoi. WWW Document IUCN Red List of Threatend Species 2019. Retrieved from http://dx.doi.org/10.2305/ IUCN.UK.2019- 2.RLTS.T125853495A125853502. en

Mokodongan, D.F. (20191). Oryzias timorensis. WWW Document IUCN Red List of Threatend Species 2019. Retrieved from http://dx.doi.org/10.2305/ IUCN.UK.2019- 2.RLTS.T90980848A90980856.en

Mokodongan, D.F. (2019m). Xenopoecilus oophorus. WWW Document IUCN Red List of Threatend Species 2019. Retrieved from http://dx.doi.org/10.2305/ IUCN.UK.2019-2.RLTS.T23121A90980873.en

Mokodongan, D.F. (2019n). Oryzias nigrimas. WWW Document IUCN Red List of Threatend Species 2019. Retrieved from https://www.iucnredlist.org/ species/pdf/90980728

Mokodongan, D.F. (2019o). Oryzias eversi. WWW Document IUCN Red List of Threatend Species 2019. Retrieved from http://dx.doi.org/10.2305/IUCN. UK.2019-2.RLTS.T90980592A90980601.en

Mokodongan, D.F. (2019p). Adrianichthys kruyti. WWW Document IUCN Red List of Threatend Species 2019. Retrieved from http://dx.doi.org/10.2305/ IUCN.UK.2019-2.RLTS.T523A90980485.en

Mokodongan, D.F. (2019q). Adrianichthys roseni. WWW Document IUCN Red List of Threatend Species 2019. Retrieved from http://dx.doi.org/10.2305/ IUCN.UK.2019-2.RLTS.T90980527A90980531.en

Mokodongan, D.F., Tanaka, R., \&Yamahira, K. (2014). A new ricefish of the genus Oryzias (Beloniformes, Adrianichthyidae) from Lake Tiu, Central Sulawesi, Indonesia. Copeia, 2014(3), 561-567.

Mokodongan, D.F., \& Yamahira, K. (2015). Origin and intra-island diversification of Sulawesi endemic Adrianichthyidae. Molecular Phylogenetics and Evolution, 93, 150-160.

Mokodongan, D.F., Montenegro, J., Mochida, K., Fujimoto, S., Ishikawa, A., Kakioka, R., ... Yamahira, K. (2018). Phylogenomics reveals habitat-associated body shape divergence in Oryzias woworae species group 
(Teleostei: Adrianichthyidae). Molecular Phylogenetics and Evolution, 118, 194-203.

Ndobe, S., Rusaini, Masyahoro, A., Serdiati, N., Madinawati, \& Moore, A.M. (2019). Meristic characters and length-weight relation of climbing perch (Anabas testudineus) from wetlands in Sigi District, Central Sulawesi, Indonesia. IOP Conference Series: Earth and Environmental Science, 370, 012001.

Nontji, A. (2016). Danau-danau alami Nusantara. Jakarta, Indonesia: Masyarakat Limnologi Indonesia.

Nursangaji, M., Rantung, E., Tokare, A., Manurung, H., Harahap, T.N., Retnowati, I., Nasution, S.R., \& Rustadi, W.C. (2014). Gerakan penyelamatan danau - Danau Poso. Jakarta, Indonesia: Kementerian Lingkungan Hidup Republik Indonesia.

NWG-EAFM. (2014). Modul penilaian indikator untuk pengelolaan perikanan dengan pendekatan ekosistem. Jakarta, Indonesia: Kementerian Kelautan dan Perikanan Republik Indonesia.

Parenti, L.R. (2008). A phylogenetic analysis and taxonomic revision of ricefishes, Oryzias and relatives (Beloniformes, Adrianichthyidae). Zoological Journal of the Linnean Society, 154(3), 494-610.

Parenti, L.R. (2011). Endemism and conservation of the native freshwater fish fauna of Sulawesi, Indonesia. Prosiding Seminar Nasional Ikan, 6, 1-10.

Parenti, L.R., \& Soeroto, B. (2004). Adrianichthys roseni and Oryzias nebulosus, two new ricefishes (Atherinomorpha: Beloniformes: Adrianichthyidae) from Lake Poso, Sulawesi, Indonesia. Ichthyological Research, 51(1), 10-19.

Pomeroy, R., Phang, K.H.W., Ramdass, K., Saad, J.M., Lokani, P., Mayo-Anda, G., ... Goby, G. (2015). Moving towards an ecosystem approach to fisheries management in the Coral Triangle region. Marine Policy, 51, 211-219.

Sadili, D., Haryono, P., Kamal, M.M., Sarmintohadi, D., \& Ramli, I. (2015). Pedoman umum restoking jenis ikan terancam punah. Indonesia, Jakarta: Kementerian Kelautan dan Perikanan.

Sari, D.K., Andriani, I., \& Yaqin, K. (2018). Histological study of the circulatory system of Sulawesi Medaka fish (Oryzias celebensis) for animal model research. Journal of Physics: Conference Series, 1028, 012008.

Schubart, C.D., \& Ng, P.K.L. (2008). A new molluscivore crab from Lake Poso confirms multiple colonization of ancient lakes in Sulawesi by freshwater crabs (Decapoda: Brachyura ). Zoological Journal of the Linnean Society, 154, 211-221.
Serdiati, N. (2019). Karakterisasi ikan padi Oryzias nigrimas (Kottelat, 1990) sebagai upaya pengelolaan ikan endemik di Danau Poso, Sulawesi Tengah. Bogor, Indonesia: Brawijaya University.

Serdiati, N., Arfiati, D., Widodo, M.S., Lelono, T.D., \& Gosari, B.A.J. (2019a). Food habits of the endemic ricefishes (Oryzias nigrimas, Kottelat 1990) in Poso Lake, Central Sulawesi, Indonesia. Russian Journal of Agricultural and Socio-Economic Sciences, 3(87), 125-130.

Serdiati, N., Arfiati, D., Widodo, M.S., Lelono, T.D., \& Toha, A.H.A. (2019b). Genetic characteristics of ricefish from Lake Poso, Central Sulawesi, Indonesia. AACL Bioflux, 12(2), 535-552.

Serdiati, N., Arfiati, D., Widodo, M.S., Lelono, T.J., Ndobe, S., \& Saranga, R. (2020). Morphological variations and phylogenetic analysis of Oryzias nigrimas Kottelat, 1990 (rice fish) from Lake Poso, Central Sulawesi, Indonesia. Biodiversitas, 21(3), 882-888.

Sutra, N., Kusumi, J., Montenegro, J., Kobayashi, H., Fujimoto, S., Masengi, K.W.A., ... Yamahira, K. (2019). Evidence for sympatric speciation in a Wallacean ancient lake. Evolution, 73(9), 1898-1915.

Vaillant, J.J., Bock, D.G., Haffner, G.D., \& Cristescu M.E. (2013). Speciation patterns and processes in the zooplankton of the ancient lakes of Sulawesi Island, Indonesia. Ecology and Evolution, 3(9), 3083-3094.

von Rintelen, T., \& Glaubrecht, M. (2006). Rapid evolution of sessility in an endemic species flock of the freshwater bivalve Corbicula from ancient lakes on Sulawesi, Indonesia. Biology Letters, 2, 73-77.

von Rintelen, K., \& Cai, Y. (2009). Radiation of endemic species flocks in ancient lakes: Systematic revision of the freshwater shrimp Caridina H. Milne Edwards, 1837 (Crustacea: Decapoda: Atyidae) from the ancient lakes of Sulawesi, Indonesia, with the description of eight new species. The Raffles Bulletin of Zoology, 57(2), 343-452.

von Rintelen, T., Stelbrink, B., Marwoto, R.M., \& Glaubrecht, M. (2014). A snail perspective on the biogeography of Sulawesi, Indonesia: Origin and intra-island dispersal of the viviparous freshwater gastropod Tylomelania. PLoS One, 9(6), e98917.

Walter, R.P., Hogan, J.D., Haffner, G.D., \& Heath, D.D. (2011). Genetic structure and connectivity among lake populations of threatened Paratherina sailfin silversides from Sulawesi, Indonesia. Conservation Genetics, 12(5), 1387-1393.

Whitten, T., Henderson, G.S., \& Mustafa, M. (2002). The ecology of Sulawesi. Singapore, Singapore: Periplus Editions (HK) Ltd. 